\title{
Risk Factors Associated with Mortality of Patients with Pelvic Fractures and Hemodynamic Instability in a Korean Trauma Center
}

\author{
Moo-Hyun Kim*, Hongjin Shim ${ }^{*}{ }^{\dagger}$, Keum Seok Bae ${ }^{*}{ }^{\dagger}$, Hoon Ryu$^{*}$, Ji Young Jang*, \\ *Department of Surgery, Yonsei University Wonju College of Medicine, \\ ${ }^{\dagger}$ Trauma Center, Wonju Severance Christian Hospital, Wonju, Korea
}

Correspondence to:
Ji Young Jang, M.D.
Department of Surgery and
Trauma Center, Wonju
Severance Christian Hospital,
Yonsei University Wonju
College of Medicine, 20 Ilsan-ro,
Wonju 26426, Korea
Tel: +82-33-741-0573
Fax: +82-33-744-6604
E-mail: jyjang@hanmail.net
ORCID:
http://orcid.org/0000-0001-6372-4194

http://orcid.org/0000-0001-6372-4194 Key Words: Pelvis, Mortality, Risk factors, Shock, Trauma centers associated with hemorrhage-induced mortality.
Purpose: The aim of this study is to evaluate treatment outcomes and mortality risks associated with hemodynamic instability caused by severe pelvic fracture in a regional trauma center.

Methods: The medical charts of 44 patients with hemodynamic instability due to pelvic fractures who were admitted to a regional trauma center from January 2014 to May 2017 were analyzed retrospectively.

Results: The mean age was 61.8 years, and the mean injury severity score was 39.1 . Twenty-six patients $(59.1 \%)$ were transferred from other hospitals, and the median time from injury to emergency room arrival was 115.5 minutes. Preperitoneal pelvic packing, pelvic angiography, and external pelvic fixation were performed in 38 patients $(86.4 \%)$ for hemostasis. The mortality rate was $52.3 \%$, and 15 patients $(34.1 \%$ ) died from hemorrhage. Logistic regression analysis showed that initial low systolic blood pressure and packed red blood cell (PRBC) requirement were independent risk factors associated with mortality. PRBC requirement for four hours and application of emergent hemostatic procedures were independent factors

Conclusion: Emergency procedures for hemostasis should be performed immediately for patients with hemodynamic instability due to pelvic fracture, and they should be transferred to a regional trauma center as soon as possible. (J Acute Care Surg 2018;8:19-24)

Received August 18, 2017, Revised September 18, 2017, Accepted September 20, 2017

Copyright $(2018$ by Korean Society of Acute Care Surgery

(c) This is an Open Access article distributed under the terms of the Creative Commons Attribution Non-Commercial License (http://creativecommons.org/licenses/by-nc/4.0) which permits unrestricted non-commercial use, distribution, and reproduction in any medium, provided the original work is properly cited.

ISSN 2288-5862(Print), ISSN 2288-9582(Online)

https://doi.org/10.17479/jacs.2018.8.1.19

\section{Introduction}

Although several hemostatic procedures have been developed in the management for patients with hemodynamic instability due to pelvic fracture, the mortality rate of these patients is still reported to be $30 \sim 60 \%$ [1-5]. Systematic approach by a multidisciplinary team and procedures for hemostasis are essential for the treatment of these patients [2]. Since the Korean government started the regional trauma center project in 2012, 17 regional trauma centers have been designated, each organized with personnel and facilities. As the only regional trauma center in Gangwon Province and having a dedicated team of trauma surgeons, Wonju Severance Christian Hospital began to treat trauma patients since 2013. A multidisciplinary approach was used for the treatment of patients with hemodynamic instability due to severe pelvic fracture, and hemostatic procedures such as pelvic 
angiography (PA), external pelvic fixation (EPF), and preperitoneal pelvic packing (PPP) were actively performed $[5,6]$. However, studies on the clinical outcome of these patients at the Korean trauma center are limited. Therefore, the purpose of this study was to evaluate treatment outcomes and risk factors associated with mortalities in patients with hemodynamic instability caused by severe pelvic fracture in a regional trauma center.

\section{Methods}

\section{Patient selection}

Between January 2014 and May 2017, 366 pelvic fracture patients were admitted at the trauma center in Wonju Severance Christian Hospital. Forty-four patients with hemodynamic instability caused by fractures were enrolled in this study. Data were obtained from the hospital's Pelvic Trauma Database, which was developed as part of the Korean Trauma Data Bank, and were analyzed retrospectively. They were reviewed for patient demographics, injury severity score (ISS), injury mechanism, time from injury to arrival at the emergency room (ER), combined injuries, type of pelvic fracture (Tile classification), hemodynamic status and laboratory findings at the time of ER arrival, packed red blood cell (PRBC) requirement, emergent hemostatic procedures (PPP, PA, and EPF), overall mortality, and hemorrhage-induced mortality. Hemodynamic instability was defined as persistent hypotension (systolic blood pressure [SBP], <90 $\mathrm{mmHg}$ ) or tachycardia (pulse rate, $>110 / \mathrm{min}$ ) despite loading $2 \mathrm{~L}$ of crystalloids and transfusion of two units of PRBCs. Patients who had severe traumatic brain injury (head Abbreviated Injury Scale [AIS] score $\geq 4$ ) or other sites with major hemorrhage were excluded from present study.

This clinical investigation was approved by the institutional review board (IRB no. CR-316111). We did not obtained written informed consent from each patient. Before data analysis, the records of patients were anonymized and de-identified. The approaches are following ordinances informed consent and enrollment expounded in guidelines.

\section{Management of hemodynamic instability due to pel- vic fracture}

Since the hospital was selected as a regional trauma center in 2012, a trauma team consisting of general, cardiothoracic, and orthopedic surgeons and emergency medicine physicians were tasked with managing trauma patients who met the criteria for the team's activation. When patients with pelvic fracture had persistent shock, a massive transfusion protocol was initiated, and extended Focused Assessment with Sonography for Trauma (eFAST) and trauma series X-ray (cervical spine lateral, chest anterior-posterior $[\mathrm{AP}]$, and pelvis $\mathrm{AP} \mathrm{X}$-ray) were performed. If fluid collection was detected in the thoracic or abdominal cavity by eFAST and severe pelvic fracture was observed on pelvis AP X-ray imaging, PPP or PA was performed before or after emergent thoracotomy or laparotomy. The order of procedures for hemostasis was determined by the trauma surgeon depending on the patient's hemodynamic status and type of associated injuries. PPP was performed on pelvic fracture patients who were hemodynamically unstable. The orthopedic surgeon will decide on the necessity of EPF.

\section{Statistical analysis}

Continuous variables were expressed as mean \pm standard deviation or median (range) and compared with Student's t-test or Mann-Whitney U test. Binary variables were compared with chi-square or Fisher's exact test. Logistic regression analysis was used to evaluate risk factors associated with mortality or hemorrhage-induced mortality, and results were expressed as odds ratios (ORs) with 95\% confidence interval (CI). Statistical significance was considered at $\mathrm{p}$-value $<0.05$. All statistical calculations were performed using IBM SPSS Statistics ver. 20.0 (IBM Co., Armonk, NY, USA).

\section{Results}

\section{Patient characteristics}

A total of 44 patients were enrolled in this study, of whom $27(61.4 \%)$ were men. The mean age was $61.8 \pm 17.5$ years. The mean ISS was $39.1 \pm 9.7$, and five patients $(11.4 \%)$ were taking anticoagulants. The most common injury mechanism was 
auto-pedestrian accident $(40.9 \%)$, followed by fall $(29.5 \%)$, motor vehicle collision $(18.2 \%)$, and motorcycle or bicycle accident $(6.8 \%)$. Twenty-six patients $(59.1 \%)$ were transferred from other hospitals, and the median time from injury to ER arrival was 115.5 (5 715) minutes. Using Tile classification for pelvic fracture, $32(72.7 \%)$ patients were classified as type B and 12 $(27.3 \%)$ as type C. The mean values of initial SBP, hemoglobin, and lactate were $67.2 \pm 9.8 \mathrm{mmHg}, 10.1 \pm 2.9 \mathrm{~g} / \mathrm{dL}$, and $5.59 \pm 3.38$ $\mathrm{mmol} / \mathrm{L}$, respectively. Thirty-eight patients (86.4\%) underwent hemostatic procedure emergently. PPP was performed in 34 patients $(77.3 \%)$, and emergent laparotomy was conducted in 11
(25.0\%). Among 15 patients (34.1\%) who underwent emergent PA, seven (46.7\%) had internal iliac artery branch embolization. Twenty-three mortalities (52.3\%) were reported, with 15 (34.1\%) caused by hemorrhage. The median duration of intensive care unit admission was seven $(0 \sim 76)$ days (Table 1$)$.

\section{Comparison between survivor and non-survivor groups in pelvic fracture patients with hemodynamic instability}

When the survivor and non-survivor groups were compared, there was no significant difference in patient characteristics (age,

Table 1. Comparison between survivors and non-survivors

\begin{tabular}{|c|c|c|c|c|}
\hline Variable & Total $(n=44)$ & Survivors $(\mathrm{n}=21)$ & Non-survivors $(\mathrm{n}=23)$ & p-value \\
\hline Age (y) & $61.8 \pm 17.5$ & $59.3 \pm 13.2$ & $64.1 \pm 20.6$ & 0.355 \\
\hline Sex, male & $27(61.4)$ & $13(61.9)$ & $14(60.9)$ & 0.944 \\
\hline Anticoagulant medication & $5(11.4)$ & $1(4.8)$ & $4(17.4)$ & $0.345^{\mathrm{a})}$ \\
\hline Injury severity score & $39.1 \pm 9.7$ & $37.7 \pm 8.0$ & $40.4 \pm 11.0$ & 0.374 \\
\hline Injury mechanism & & & & $0.825^{\mathrm{a})}$ \\
\hline Auto-pedestrian accident & $18(40.9)$ & $8(38.1)$ & $10(43.5)$ & \\
\hline Motor vehicle collision & $8(18.2)$ & $4(19.0)$ & $4(17.4)$ & \\
\hline Fall & $13(29.5)$ & $8(38.1)$ & $5(21.7)$ & \\
\hline Crush & $2(4.5)$ & 0 & $2(8.7)$ & \\
\hline Motorcycle and bicycle & $3(6.8)$ & $1(4.8)$ & $2(8.7)$ & \\
\hline Transfer from another hospital & $26(59.1)$ & $12(57.1)$ & $14(60.9)$ & 0.802 \\
\hline Time from injury to ER arrival (min) & $115.5(5 \sim 715)$ & $105(21 \sim 370)$ & $126(5 \sim 715)$ & 0.537 \\
\hline Trauma resuscitation room stay (min) & $108 \pm 62$ & $113 \pm 74$ & $104 \pm 50$ & 0.639 \\
\hline Open fracture & $2(4.5)$ & 0 & $2(8.7)$ & $0.489^{\mathrm{b})}$ \\
\hline Pelvic fracture type & & & & 0.853 \\
\hline Tile classification B & $32(72.7)$ & $15(71.4)$ & $17(73.9)$ & \\
\hline Tile classification $\mathrm{C}$ & $12(27.3)$ & $6(28.6)$ & $6(26.1)$ & \\
\hline Combined injury & $39(88.6)$ & $19(90.5)$ & $20(87.0)$ & $1.000^{\mathrm{a})}$ \\
\hline Initial SBP $(\mathrm{mmHg})$ & $67.2 \pm 9.8$ & $70.6 \pm 9.8$ & $64.0 \pm 8.8$ & $0.025^{\mathrm{b})}$ \\
\hline Initial hemoglobin $(\mathrm{g} / \mathrm{dL})$ & $10.1 \pm 2.9$ & $10.2 \pm 2.2$ & $10.1 \pm 3.4$ & 0.833 \\
\hline Initial lactate $(\mathrm{mmol} / \mathrm{L})$ & $5.59 \pm 3.38$ & $4.12 \pm 2.17$ & $6.92 \pm 3.77$ & $0.004^{\mathrm{b})}$ \\
\hline PRBC requirement for $4 \mathrm{~h}$ (unit) & $11.9 \pm 9.7$ & $8.7 \pm 5.2$ & $14.8 \pm 11.9$ & 0.031 \\
\hline PRBC requirement (unit) & $17.4 \pm 12.6$ & $12.9 \pm 6.3$ & $21.5 \pm 15.3$ & $0.019^{\mathrm{b})}$ \\
\hline Preperitoneal pelvic packing & $34(77.3)$ & $7(33.3)$ & $16(69.6)$ & $0.287^{\mathrm{a})}$ \\
\hline Laparotomy & $11(25.0)$ & $3(14.3)$ & $8(34.8)$ & 0.117 \\
\hline Emergent external fixation & $9(20.5)$ & $6(28.6)$ & $3(13.0)$ & $0.272^{\mathrm{a})}$ \\
\hline ORIF & $13(29.5)$ & $11(52.4)$ & $2(8.7)$ & $0.002^{\mathrm{b})}$ \\
\hline Emergent pelvic angiography & $15(34.1)$ & $8(38.1)$ & $7(30.4)$ & 0.592 \\
\hline Emergent hemostatic procedure & $38(86.4)$ & $21(100)$ & $17(73.9)$ & $0.022^{\mathrm{b})}$ \\
\hline ICU stay (day) & $7(0 \sim 76)$ & $14(4 \sim 76)$ & $1(0 \sim 39)$ & $0.002^{\mathrm{b})}$ \\
\hline
\end{tabular}

Values are presented as mean \pm standard deviation or number (\%).

ER: emergency room, SBP: systolic blood pressure, PRBC: packed red blood cell, ORIF: open reduction and internal fixation, ICU: intensive care unit.

${ }^{a)}$ Results of Fisher's exact test; ${ }^{\text {b) }}$ The data were statistically significant. 
gender, and history of anticoagulant use), injury mechanism, ISS, rate of transfer from another hospital, time from injury to ER arrival, combined injury, and pelvic fracture type. The non-survivor group had significantly lower initial SBP (64.0 28.8 vs. $70.6 \pm 9.8 \mathrm{mmHg}, \mathrm{p}=0.025)$ and higher initial lactate $(6.92 \pm 3.77$ vs. $4.12 \pm 2.17 \mathrm{mmol} / \mathrm{L}, \mathrm{p}=0.004)$ than the survivor group. The total amount of transfusion $(21.5 \pm 15.3$ vs. $12.9 \pm 6.3$ units, $\mathrm{p}=0.019)$ and amount of blood transfusion for 4 hours (14.8 \pm 11.9 vs. $8.7 \pm 5.2$ units, $\mathrm{p}=0.031$ ) were significantly higher in the non-survivor than in the survivor group. Although there was no difference in the frequencies of PPP, PA, and EPF between the two groups respectively, the overall frequency of hemostatic procedures was significantly higher in the survivor group $(100 \%$ vs. $73.9 \%, \mathrm{p}=0.022$ ) (Table 1$)$.

\section{Multivariate analysis of factors associated with mor- tality in pelvic fracture patients with hemodynamic in- stability}

Factors such as "emergent hemostatic procedure," "initial SBP," "initial lactate," and "PRBC requirement" were subjected to multivariate analysis with logistic regression model. The results showed that initial SBP (OR, 0.902; 95\% CI, 0.825 0.987; $\mathrm{p}=0.024)$ and $\mathrm{PRBC}$ requirement $(\mathrm{OR}, 1.112$; 95\% CI, 1.008 1.228; $\mathrm{p}=0.034)$ were independent factors associated with mortality in pelvic fracture patients with hemodynamic instability (Table 2).

Table 2. Multivariate analysis of factors associated with mortality in pelvic fracture patients with hemodynamic instability

\begin{tabular}{lcl}
\hline \multirow{2}{*}{\multicolumn{1}{c}{ Variable }} & \multicolumn{2}{c}{ Risk factors for mortality } \\
\cline { 2 - 3 } & OR $(95 \% \mathrm{CI})$ & $\mathrm{p}$-value \\
\hline Emergent hemostatic procedure & Undefined & 0.999 \\
Initial systolic blood pressure & $0.902(0.825 \sim 0.987)$ & $0.024^{\mathrm{a})}$ \\
Initial lactate & $1.142(0.836 \sim 1.562)$ & 0.404 \\
PRBC requirement & $1.112(1.008 \sim 1.228)$ & $0.034^{\mathrm{a})}$ \\
\hline
\end{tabular}

OR: odds ratio, CI: confidence interval, PRBC: packed red blood cell.

a) The data were statistically significant.

\section{Multivariate analysis of factors associated with hem- orrhage-induced mortality in pelvic fracture patients with hemodynamic instability}

When 15 patients with hemorrhagic mortality were compared with patients without hemorrhage mortality, there were significant differences in "application of PPP” (53.3\% vs. 89.7\%, p=0.011), "application of emergent procedures" (66.7\% vs. $96.6 \%, \mathrm{p}=0.013)$, "initial lactate" (7.33 vs. $4.68 \mathrm{mmol} / \mathrm{L}, \mathrm{p}=0.012)$, and "PRBC requirement for 4 hours" (17.1 vs. 9.2 units, $\mathrm{p}=0.004)$. Logistic regression analysis showed that "emergent hemostatic procedure" (OR, 0.045; 95\% CI, 0.004 0.489; $\mathrm{p}=0.011$ ) and "PRBC requirement for 4 hours" (OR, 1.129; 95\% CI, 1.022 1.247; $\mathrm{p}=0.017)$ were independent factors associated with hemorrhage-induced mortality (Table 3).

\section{Discussion}

In this study, initial SBP and PRBC requirements were identified as factors associated with overall mortality. Previous studies showed that predictors of mortality for pelvic ring fracture were age, ISS, pelvic ring instability, open fracture, rectal injury, combined head injury, units of PRBC transfused, and admission base deficit [7-9]. However, in our study, patients' age, ISS, and pelvic fracture type were not independent factors related with mortality. It might have been caused by the exclusion of patients with severe head trauma (AIS $\geq 4$ ) and weak statistical power

Table 3. Multivariate analysis of factors associated with hemorrhagic mortality in pelvic fracture patients with hemodynamic instability

\begin{tabular}{lll}
\hline \multirow{2}{*}{ Variable } & \multicolumn{2}{c}{$\begin{array}{c}\text { Risk factors for mortality } \\
\text { due to hemorrhage }\end{array}$} \\
\cline { 2 - 3 } & OR $(95 \%$ CI $)$ & p-value \\
\hline Preperitoneal pelvic packing & $0.191(0.024 \sim 1.523)$ & 0.118 \\
Emergent hemostatic procedure & $0.045(0.004 \sim 0.489)$ & $0.011^{\mathrm{a})}$ \\
Initial lactate & $1.110(0.826 \sim 1.492)$ & 0.489 \\
PRBC requirement for $4 \mathrm{~h}$ & $1.129(1.022 \sim 1.247)$ & $0.017^{\mathrm{a})}$ \\
\hline
\end{tabular}

The logistic regression model includes "preperitoneal pelvic packing," "emergent hemostatic procedure," "initial lactate," and "PRBC requirement for 4 hours."

OR: odds ratio, CI: confidence interval, PRBC: packed red blood cell.

a) The data were statistically significant. 
in multivariate analysis due to the small number of enrolled patients.

There are several reasons for the high overall mortality (52.3\%), which was compared with previous studies. First, although patients who were admitted after the establishment of a trauma center were enrolled in this study, the median time from injury to ER arrival was 115.5 minutes, which was still a long duration. This seems to be due to the deterioration of the patient's condition upon arrival at the trauma center, with about $60 \%$ of the patients being transferred from other hospitals. Second, despite the formation of a trauma team in our hospital in 2014, definite management protocols for pelvic fracture patients with shock has still not been established. Biffl et al. [2] reported that a multidisciplinary clinical pathway, decided by trauma surgeons and orthopedic traumatologists, improved patient survival. Because of incomplete consensus on the order and method of hemostatic procedures among trauma surgeons and the lack of orthopedic surgeons with a traumatology background, there were difficulties in decision-making at our hospital. Third, the mean age of enrolled patients in our study was high. Although a recent multi-institutional trial by the American Association for the Surgery of Trauma reported that the mortality rate of patients with hemodynamic instability due to severe pelvic fracture was $32.0 \%$; the mean age and median ISS of patients enrolled in that study were 44.0 and 28 years, respectively, which were lower than that in our study [1].

Fifteen patients $(34.1 \%)$ died of acute hemorrhage, and independent factors associated with mortality were PRBC requirement for 4 hours and emergent hemostatic procedure. Generally, PPP, PA, and EPF were performed as emergent procedures to control hemorrhage due to pelvic fracture. Several studies showed that PPP provided effective hemostasis and improved clinical outcomes in pelvic fracture patients with hemodynamic instability [6,10-12].

Burlew et al. [13] reported that the application of the Denver Health Protocol for unstable pelvic fracture management, including PPP and concurrent EPF prior to PA, has resulted in $8 \%$ overall mortality and $21 \%$ mortality in high-risk patients. The pelvic trauma management algorithm, published in 2017 by the World Society of Emergency Surgery, recommended EPF or PA or resuscitative endovascular balloon occlusion of the aorta as a complementary procedure after PPP was used for initial hemostasis [14]. Our data showed that PPP was significant for hemorrhage-induced mortality in univariate analysis, but not in the multivariate analysis. This suggests that PPP alone was not sufficient to prevent hemorrhage-induced mortality in these patients, and any hemostatic procedure should be performed without delay in accordance with certain protocols.

Hemorrhage in pelvic fracture patients is mainly caused by bleeding from the fractured bone and injury of internal iliac vessels and their branches [5]. In case of disruption of the posterior arch of the pelvic ring (sacroiliac joint destruction), migration of hemorrhage from pelvic cavity to retroperitoneal cavity deprives tamponade ability of pelvic cavity [15-18]. In our study, the time from injury to ER arrival of 26 patients who were transferred from another hospital was long (213 minutes). Although patients did not have low blood pressure initially in many cases, they were transferred after identification of hypotension or abdominal distension due to pelvic fracture was delayed. If patients have hemodynamic instability with low possibility of chest or abdominal injury, hemorrhage due to pelvic injury should be suspected, and pelvic fracture type and occurrence of sacroiliac joint injury should be evaluated using a portable X-ray machine [19]. It was known that the most sensitive markers to identify hemorrhage were serum lactate and base deficit by arterial blood gas analysis; moreover, hemoglobin level and hematocrit did not represent acute hemorrhage in pelvic fracture patients [20]. In the present study, patients with hemorrhage-induced mortality had significantly higher level of serum lactate than survivors. Taken together, if patients with low possibility of chest and abdominal injury have severe pelvic fracture and high serum lactate, ongoing hemorrhage due to pelvic fracture should be suspected, and they should be transferred quickly to a regional trauma center where hemostatic procedures such as PPP, EPF, and PA are performed. In addition, continuous education should be provided for emergency physicians at hospitals that transfer patients to a regional trauma center.

The major limitations of our study are its small size and retrospective nature, and that patients with pelvic fractures but no hemodynamic instability were not analyzed. In spite of these 
limitations, this study is still meaningful as it provides information on the outcomes and factors related to death in pelvic fracture patients with hemodynamic instability in a Korean regional trauma center. However, a large-scale and multicenter study with Korean trauma data bank analysis will be needed in the future.

\section{Acknowledgments}

We thank the trauma team and the staff of the trauma intensive care unit of Wonju Severance Christian hospital for their devotion and affection for patients.

\section{Conflicts of Interest}

No potential conflict of interest relevant to this article was reported.

\section{References}

1. Costantini TW, Coimbra R, Holcomb JB, Podbielski JM, Catalano R, Blackburn A, et al. Current management of hemorrhage from severe pelvic fractures: Results of an American Association for the Surgery of Trauma multi-institutional trial. J Trauma Acute Care Surg 2016;80:717-23; discussion 723-5.

2. Biffl WL, Smith WR, Moore EE, Gonzalez RJ, Morgan SJ, Hennessey $T$, et al. Evolution of a multidisciplinary clinical pathway for the management of unstable patients with pelvic fractures. Ann Surg 2001;233:843-50.

3. Cothren CC, Osborn PM, Moore EE, Morgan SJ, Johnson JL, Smith WR. Preperitonal pelvic packing for hemodynamically unstable pelvic fractures: a paradigm shift. J Trauma 2007;62:834-9; discussion 839-42.

4. Eastridge BJ, Starr A, Minei JP, O'Keefe GE, Scalea TM. The importance of fracture pattern in guiding therapeutic decision-making in patients with hemorrhagic shock and pelvic ring disruptions. J Trauma 2002;53:446-50; discussion 450-1.

5. Suzuki T, Smith WR, Moore EE. Pelvic packing or angiography: competitive or complementary? Injury 2009;40:343-53.

6. Jang JY, Shim H, Jung PY, Kim S, Bae KS. Preperitoneal pelvic packing in patients with hemodynamic instability due to severe pelvic fracture: early experience in a Korean trauma center. Scand J Trauma Resusc Emerg Med 2016;24:3.

7. Ojodu I, Pohlemann T, Hopp S, Rollmann MF, Holstein JH, Herath SC. Predictors of mortality for complex fractures of the pelvic ring in the elderly: a twelve-year review from a German level I trauma center. Injury 2015;46:1996-8.

8. Grotz MR, Allami MK, Harwood P, Pape HC, Krettek C, Giannoudis PV. Open pelvic fractures: epidemiology, current concepts of management and outcome. Injury 2005;36:1-13.

9. Perkins ZB, Maytham GD, Koers L, Bates P, Brohi K, Tai NR. Impact on outcome of a targeted performance improvement programme in haemodynamically unstable patients with a pelvic fracture. Bone Joint J 2014;96-B:1090-7.

10. Li Q, Dong J, Yang Y, Wang G, Wang Y, Liu P, et al. Retroperitoneal packing or angioembolization for haemorrhage control of pelvic fractures--Quasi-randomized clinical trial of 56 haemodynamically unstable patients with Injury Severity Score $\geq 33$. Injury 2016;47:395-401.

11. Osborn PM, Smith WR, Moore EE, Cothren CC, Morgan SJ, Williams AE, et al. Direct retroperitoneal pelvic packing versus pelvic angiography: A comparison of two management protocols for haemodynamically unstable pelvic fractures. Injury 2009;40:54-60.

12. Tai DK, Li WH, Lee KY, Cheng M, Lee KB, Tang LF, et al. Retroperitoneal pelvic packing in the management of hemodynamically unstable pelvic fractures: a level I trauma center experience. J Trauma 2011;71:E79-86.

13. Burlew CC, Moore EE, Stahel PF, Geddes AE, Wagenaar AE, Pieracci FM, et al. Preperitoneal pelvic packing reduces mortality in patients with life-threatening hemorrhage due to unstable pelvic fractures. J Trauma Acute Care Surg 2017;82: 233-42.

14. Coccolini F, Stahel PF, Montori G, Biffl W, Horer TM, Catena F, et al. Pelvic trauma: WSES classification and guidelines. World J Emerg Surg 2017;12:5.

15. Ghanayem AJ, Wilber JH, Lieberman JM, Motta AO. The effect of laparotomy and external fixator stabilization on pelvic volume in an unstable pelvic injury. J Trauma 1995;38:396-400; discussion 400-1.

16. Moss MC, Bircher MD. Volume changes within the true pelvis during disruption of the pelvic ring--where does the haemorrhage go? Injury 1996;27 Suppl 1:S-A21-3.

17. Durkin A, Sagi HC, Durham R, Flint L. Contemporary management of pelvic fractures. Am J Surg 2006;192:211-23.

18. Grimm MR, Vrahas MS, Thomas KA. Pressure-volume characteristics of the intact and disrupted pelvic retroperitoneum. J Trauma 1998;44:454-9.

19. Cullinane DC, Schiller HJ, Zielinski MD, Bilaniuk JW, Collier BR, Como J, et al. Eastern Association for the Surgery of Trauma practice management guidelines for hemorrhage in pelvic fracture--update and systematic review. J Trauma 2011;71:1850-68.

20. Rossaint R, Bouillon B, Cerny V, Coats TJ, Duranteau J, Fernández-Mondéjar E, et al. Management of bleeding following major trauma: an updated European guideline. Crit Care 2010;14:R52. 